\title{
THE NEED FOR A NEW CLASSIFICATION OF DOUBLE HYPERGEOMETRIC SERIES
}

\author{
B. C. CARLSON
}

\begin{abstract}
An elementary proof is given of a linear transformation which changes a particular double hypergeometric series of order two into a series of order three. A similar transformation holds for a particular series of arbitrary order in two or more variables. The change in order provides new evidence, the most compelling to date, that the order of such a series is not a fundamental property. This conclusion undermines the accepted classification of hypergeometric series in more than one variable.
\end{abstract}

1. Introduction. The double power series

$$
\sum_{m, n=0}^{\infty} A(m, n) x^{m} y^{n}
$$

is called a hypergeometric series, according to the definition introduced by Horn [5] in 1889, if the two quotients

$$
\frac{A(m+1, n)}{A(m, n)}=f(m, n), \quad \frac{A(m, n+1)}{A(m, n)}=g(m, n)
$$

are rational functions of $m$ and $n$. That is,

$$
f(m, n)=\frac{F(m, n)}{F^{\prime}(m, n)}, \quad g(m, n)=\frac{G(m, n)}{G^{\prime}(m, n)},
$$

where $F, F^{\prime}, G, G^{\prime}$ are polynomials in $m$ and $n$. [Horn assumed that $A(m, n)$ contains a factor $(m ! n !)^{-1}$ even if it is compensated by a factor $m ! n !$. Hence $F^{\prime}$ contains a factor $m+1$, possibly in common with $F$, but $F$ and $F^{\prime}$ have no other common factors. Similar remarks apply to $G, G^{\prime}$, and $n+1$. The possibility of exceptional common factors may be ignored in the remainder of this paper.] If $F, F^{\prime}, G, G^{\prime}$ have respective degrees $p, p^{\prime}, q, q^{\prime}$, the largest of these four integers is defined to be the order of the series.

Series of order one are reducible to series in a single variable. The class of series of order two has been studied in some detail; there are 14 complete series for which $p=p^{\prime}=q=q^{\prime}=2$ (including the four well-known Appell series) and 20 confluent series. A survey of the theory, with references, is given in $[4, \S \S 5.7-5.14]$, where the 34 series of order two are listed together with the systems of partial differential equations which they satisfy.

A defect in this classification by order was noticed in 1948 by Erdélyi [3, p. 380], who found but did not record a linear transformation connecting two

Received by the editors March 28, 1975.

AMS (MOS) subject classifications (1970). Primary 33A30. 
series of second order with one series of third order. Recently Carlson [1] found a quadratic transformation connecting one series of second order with one series of third order and subsequently [2] deduced from this several more quadratic transformations of the same type. Since each of these examples involves some element of complexity in the result or the proof or both, it seems worthwhile to give a self-contained elementary proof of a simple linear transformation connecting one series of second order with one series of third order. This example of a change in order seems the most compelling evidence yet found that the notion of order has no fundamental significance and that the theory of double hypergeometric series needs a new starting point.

2. Example of a change in order. Let $a, b, b^{\prime}, c$ be real and strictly positive, and assume $a \neq c, b+b^{\prime} \neq c, b \neq 1$, and $b^{\prime} \neq 1$. Define $(a)_{0}=1$ and $(a)_{m}=a(a+1)(a+2) \cdots(a+m-1)$ if $m \geqslant 1$. Let $x$ and $y$ be complex numbers such that $|x-y|+|y|<1$. The transformation to be proved is

$$
\sum_{m, n=0}^{\infty} \frac{(a)_{m+n}(b)_{m}\left(b^{\prime}\right)_{n}}{(c)_{m+n} m ! n !} x^{m} y^{n}=\sum_{m, n=0}^{\infty} \frac{(a)_{m+n}\left(b+b^{\prime}\right)_{m+n}(b)_{m}}{(c)_{m+n}\left(b+b^{\prime}\right)_{m} m ! n !}(x-y)^{m} y^{n}
$$

For the left side the polynomials defined by (2) and (3) are

$$
\begin{aligned}
F(m, n) & =(a+m+n)(b+m), & G(m, n) & =(a+m+n)\left(b^{\prime}+n\right), \\
F^{\prime}(m, n) & =(c+m+n)(1+m), & G^{\prime}(m, n) & =(c+m+n)(1+n) .
\end{aligned}
$$

Hence $p=p^{\prime}=q=q^{\prime}=2$, and the left side is a complete series of order two. (It is Appell's series $F_{1}$.) For the right side,

$$
\begin{aligned}
F(m, n) & =(a+m+n)\left(b+b^{\prime}+m+n\right)(b+m), \\
F^{\prime}(m, n) & =(c+m+n)\left(b+b^{\prime}+m\right)(1+m), \\
G(m, n) & =(a+m+n)\left(b+b^{\prime}+m+n\right), \\
G^{\prime}(m, n) & =(c+m+n)(1+n) .
\end{aligned}
$$

Here $p=p^{\prime}=3$ and $q=q^{\prime}=2$, and the right side of (4) is a series of order three.

To prove (4) we show first that both double series converge absolutely. Since $(b)_{m} \leqslant\left(b+b^{\prime}\right)_{m}$ absolute convergence of the series on the right side is guaranteed by the convergence of a series of positive terms,

$$
\sum_{m, n=0}^{\infty} \frac{(a)_{m+n}\left(b+b^{\prime}\right)_{m+n}}{(c)_{m+n} m ! n !}|x-y|^{m}|y|^{n}=\sum_{N=0}^{\infty} \frac{(a)_{N}\left(b+b^{\prime}\right)_{N}}{(c)_{N} N !}(|x-y|+|y|)^{N} .
$$

The last series converges by the ratio test.

In dealing with the left side of (4), and again later, we shall use a form of Vandermonde's theorem,

$$
\sum_{m+n=N} \frac{(b)_{m}\left(b^{\prime}\right)_{n}}{m ! n !}=\frac{\left(b+b^{\prime}\right)_{N}}{N !},
$$

where the summation extends over all nonnegative integers $m$ and $n$ whose sum is $N$. [The proof consists in observing that the right side is the coefficient 
of $t^{N}$ in $(1-t)^{-b-b^{\prime}}$ while the left side is the same coefficient in the product of $(1-t)^{-b}$ and $(1-t)^{-b^{\prime}}$.] If $X=\max \{|x|,|y|\}$ then $X<1$, because $|y| \leqslant|x-y|+|y|<1$ and $|x|=|x-y+y| \leqslant|x-y|+|y|<1$. The absolute convergence of the series on the left side of (4) is guaranteed by the convergence of

$$
\begin{aligned}
\sum_{m, n=0}^{\infty} \frac{(a)_{m+n}(b)_{m}\left(b^{\prime}\right)_{n}}{(c)_{m+n} m ! n !} X^{m+n} & =\sum_{N=0}^{\infty} \frac{(a)_{N}}{(c)_{N}} X^{N} \sum_{m+n=N} \frac{(b)_{m}\left(b^{\prime}\right)_{n}}{m ! n !} \\
& =\sum_{N=0}^{\infty} \frac{(a)_{N}\left(b+b^{\prime}\right)_{N}}{(c)_{N} N !} X^{N}
\end{aligned}
$$

The last series converges by the ratio test.

Since both series in (4) converge absolutely, we may sum them in the order indicated by $\sum_{N=0}^{\infty} \sum_{m+n=N}$. Therefore, it suffices to prove that

(8) $\sum_{m+n=N} \frac{(b)_{m}\left(b^{\prime}\right)_{n}}{m ! n !} x^{m} y^{n}=\left(b+b^{\prime}\right)_{N} \sum_{m+n=N} \frac{(b)_{m}}{\left(b+b^{\prime}\right)_{m} m ! n !}(x-y)^{m} y^{n}$.

Denoting the left side by $S$, we use the binomial theorem in writing

$$
\begin{aligned}
S & =\sum_{n=0}^{N} \frac{(b)_{N-n}\left(b^{\prime}\right)_{n}}{(N-n) ! n !}(x-y+y)^{N-n} y^{n} \\
& =\sum_{n=0}^{N} \frac{(b)_{N-n}\left(b^{\prime}\right)_{n}}{(N-n) ! n !} \sum_{m=0}^{N-n}\left(\begin{array}{c}
N-n \\
m
\end{array}\right)(x-y)^{m} y^{N-m} .
\end{aligned}
$$

A change in the order of summation gives

$$
S=\sum_{m=0}^{N} \frac{(x-y)^{m} y^{N-m}}{m !} \sum_{n=0}^{N-m} \frac{(b)_{N-n}\left(b^{\prime}\right)_{n}}{(N-m-n) ! n !} .
$$

Using first the identity

$$
\begin{aligned}
(b)_{N-n} & =b(b+1) \cdots(b+m-1)(b+m) \cdots(b+N-n-1) \\
& =(b)_{m}(b+m)_{N-m-n}
\end{aligned}
$$

and subsequently Vandermonde's theorem (7), we can evaluate

$$
\begin{aligned}
\sum_{n=0}^{N-m} \frac{(b)_{N-n}\left(b^{\prime}\right)_{n}}{(N-m-n) ! n !} & =(b)_{m} \sum_{n=0}^{N-m} \frac{(b+m)_{N-m-n}\left(b^{\prime}\right)_{n}}{(N-m-n) ! n !} \\
& =\frac{(b)_{m}\left(b+b^{\prime}+m\right)_{N-m}}{(N-m) !}=\frac{(b)_{m}\left(b+b^{\prime}\right)_{N}}{(N-m) !\left(b+b^{\prime}\right)_{m}} .
\end{aligned}
$$

Substituting (11) in (10) gives

$$
S=\left(b+b^{\prime}\right)_{N} \sum_{m=0}^{N} \frac{(b)_{m}}{\left(b+b^{\prime}\right)_{m} m !(N-m) !}(x-y)^{m} y^{N-m} .
$$

This equals the right side of (8), and so (8) and (4) are proved. 
Nothing in the method of proof is affected by deleting on both sides of (4) the ratio $(a)_{m+n} /(c)_{m+n}$ nor by inserting on both sides of (4) one or more ratios of the form $\left(a^{\prime}\right)_{m+n} /\left(c^{\prime}\right)_{m+n}$, where $a^{\prime}$ and $c^{\prime}$ are real and strictly positive. For every positive integer $M$, therefore, we have proved a linear transformation connecting one series of order $M$ with one series of order $M+1$.

This result can readily be extended to series in several variables. The form of the general case is made sufficiently clear by giving the case of three variables and $M=2$. If $|x-z|+|y-z|+|z|<1$ then

$$
\begin{aligned}
& \sum_{m, n, p=0}^{\infty} \frac{(a)_{m+n+p}(b)_{m}\left(b^{\prime}\right)_{n}\left(b^{\prime \prime}\right)_{p}}{(c)_{m+n+p} m ! n ! p !} x^{m} y^{n} z^{p} \\
& =\sum_{m, n, p=0}^{\infty} \frac{(a)_{m+n+p}\left(b+b^{\prime}+b^{\prime \prime}\right)_{m+n+p}(b)_{m}\left(b^{\prime}\right)_{n}}{(c)_{m+n+p}\left(b+b^{\prime}+b^{\prime \prime}\right)_{m+n} m ! n ! p !}(x-z)^{m}(y-z)^{n} z^{p}
\end{aligned}
$$

\section{REFERENCES}

1. B. C. Carlson, Appell's function $F_{4}$ as a double average, SIAM J. Math. Anal. 6 (1975), 960-965.

2. — Quadratic transformations of Appell functions, SIAM J. Math. Anal. 7 (1976).

3. A. Erdélyi, Transformations of hypergeometric functions of two variables, Proc. Roy. Soc. Edinburgh Sect. A 62(1948), 378-385. MR 10, 115.

4. A. Erdélyi, W. Magnus, F. Oberhettinger and F. G. Tricomi, Higher transcendental functions. Vols. I, II, McGraw-Hill, New York, 1953. MR 15, 419.

5. : Horn, Ueber die Convergenz der hypergeometrischen Reihen zweier und dreier Veränderlichen, Math. Ann. 34 (1889), 544-600.

6. - Hypergeometrische Funktionen zweier Veränderlichen, Math. Ann. 105(1931), 381-407.

ames Laboratory-ERda and Department of Mathematics and Physics, Iowa State UNIVERSITY, AMES, IOWA 50011 